\title{
ON THE HAUSDORFF DIMENSION OF THE JULIA SET OF A REGULARLY GROWING ENTIRE FUNCTION
}

\author{
WALTER BERGWEILER AND BOGUSEAWA KARPIŃSKA
}

\begin{abstract}
We show that if the growth of a transcendental entire function $f$ is sufficiently regular, then the Julia set and the escaping set of $f$ have Hausdorff dimension 2.
\end{abstract}

\section{INTRODUCTION AND RESUltS}

The Julia set $J(f)$ of an entire function $f$ is defined as the set of all points in the plane where the iterates $f^{n}$ of $f$ do not form a normal family. Denote by $\operatorname{dim} E$ the Hausdorff dimension and by area $E$ the Lebesgue measure of a subset $E$ of the plane.

McMullen [13] proved that $\operatorname{dim} J\left(\lambda e^{z}\right)=2$ for $\lambda \in \mathbb{C}, \lambda \neq 0$. He also proved that area $J(\sin (\alpha z+\beta))>0$ and hence $\operatorname{dim} J(\sin (\alpha z+\beta))=2$ for $\alpha, \beta, \in \mathbb{C}, \alpha \neq 0$. In the proofs, he first showed that these results hold if the Julia set $J(f)$ is replaced by the escaping set $I(f)=\left\{z \in \mathbb{C}: f^{n}(z) \rightarrow \infty\right\}$, and then he showed that $I(f) \subset J(f)$ for the functions $f$ considered.

McMullen's results have been extended to various classes of entire functions; see [1, 2, 5, 17, 20]. All these extensions concern the Eremenko-Lyubich class $B$ which consists of all entire functions for which the set of finite asymptotic values and critical values is bounded. Here we only mention the result of Barański [2] and Schubert [17] which says that $\operatorname{dim} J(f)=2$ if $f \in B$ and if $f$ has finite order. Recall that the order $\rho(f)$ of an entire function $f$ is defined by

$$
\rho(f)=\limsup _{r \rightarrow \infty} \frac{\log \log M(r, f)}{\log r}, \quad \text { where } \quad M(r, f)=\max _{|z|=r}|f(z)| .
$$

One advantage of working with the class $B$ is that $I(f) \subset J(f)$ for $f \in B$ by a result of Eremenko and Lyubich [6, Theorem 1] so that the second part of McMullen's argument carries over directly to this class.

Eremenko and Lyubich prove their result that $I(f) \subset J(f)$ by introducing a logarithmic change of variable to the subject. This logarithmic change of variable has become a very powerful tool in transcendental dynamics and it is the main reason why a considerable amount of research has been devoted to the class $B$. This includes results on Hausdorff dimension (e.g., [3, 18]), but also on various other topics (e.g., [15, 16]).

The purpose of this paper is to obtain a result on the Hausdorff dimension of the Julia set for entire functions which do not belong to the Eremenko-Lyubich class and for

Date: November 15, 2018.

1991 Mathematics Subject Classification. 37F10 (primary), 30D05, 30D15 (secondary).

The authors were supported by the EU Research Training Network CODY. The first author was also supported by the the Deutsche Forschungsgemeinschaft, Be 1508/7-1, the ESF Research Networking Programme HCAA and the G.I.F., the German-Israeli Foundation for Scientific Research and Development, Grant G-809-234.6/2003. The second author was also supported by Polish MNiSW Grant N N201 022233 and PW Grant 504G 11200011000. 
which the logarithmic change of variable therefore is not available. We consider functions which grow regularly in a certain sense. More precisely, we will be concerned with entire functions $f$ for which there exist $A, B, C, r_{0}>1$ such that

$$
A \log M(r, f) \leq \log M(C r, f) \leq B \log M(r, f) \quad \text { for } r \geq r_{0} .
$$

Our main result is the following.

Theorem 1.1. Let $f$ be an entire function satisfying (1.1). Then $\operatorname{dim}(I(f) \cap J(f))=2$.

We note that the hypothesis (1.1) is satisfied if there exists $c_{1}, c_{2}, \rho>0$ such that

$$
c_{1} r^{\rho} \leq \log M(r, f) \leq c_{2} r^{\rho}
$$

for large $r$ and thus in particular if there exists $c, \rho>0$ such that

$$
\log M(r, f) \sim c r^{\rho}
$$

as $r \rightarrow \infty$. It is classical that (1.3) holds for transcendental entire functions which satisfy an algebraic differential equation of first order [22, Section IV.6] or a linear differential equation whose coefficients are rational functions [22, Section IV.5]. As another example we mention Poincaré functions associated to repelling fixed points of polynomials or, more generally, transcendental entire solutions of the functional equation $f(s z)=P(f(z), z)$ where $|s|>1$ and $P$ is a polynomial in two variables with $\operatorname{deg}_{f} P \geq 2$. A solution $f$ of such an equation satisfies (1.2) for large $r$; see [22, Section II.8]. Finally we note that (1.3) is satisfied by functions of completely regular growth in the sense of Pfluger; see [12, Section 3] for a thorough treatment of this class of functions.

We note that the condition (1.1) does not imply that $I(f) \subset J(f)$. For example, for the function $f(z)=z+1+e^{-z}$ already considered by Fatou [7, Exemple 1, p. 358] we have $\log M(r, f) \sim r$ while $\{z \in \mathbb{C}: \operatorname{Re} z>0\} \subset I(f) \backslash J(f)$. We will further discuss the condition (1.1) in section 2.1,

Among the tools used in the proof of Theorem 1.1 are the Ahlfors islands theorem (see Lemma 4.3 below) and a result on the Hausdorff dimension of the intersection of nested sets due to McMullen (see Lemma 4.4 below). In addition, the proof requires some careful estimates of the logarithmic derivative of $f$. As these estimates of the logarithmic derivative may be of independent interest, we include them in this introductory section.

For $\alpha_{1}, \alpha_{2}, q, \lambda \geq 0$ we consider the set $T\left(f, \alpha_{1}, \alpha_{2}, q, \lambda\right)$ consisting of all $z \in \mathbb{C}$ for which

$$
\begin{gathered}
\alpha_{1} \log M(|z|, f) \leq\left|\frac{z f^{\prime}(z)}{f(z)}\right| \leq \alpha_{2} \log M(|z|, f), \\
|f(z)| \geq|z|^{q}
\end{gathered}
$$

and

$$
\left|\frac{\zeta f^{\prime}(\zeta)}{f(\zeta)}\right| \leq \alpha_{2} \log M(|\zeta|, f) \quad \text { for } \quad|\zeta-z| \leq \lambda \frac{|z|}{\log M(|z|, f)}
$$

Of course, the right inequality of (1.4) is a special case of (1.6).

For $R>0$ we put $A(R)=\{z \in \mathbb{C}: R \leq|z| \leq 2 R\}$. For measurable sets $X, Y \subset \mathbb{C}$ the density of $X$ in $Y$ is defined by

$$
\operatorname{dens}(X, Y)=\frac{\operatorname{area}(X \cap Y)}{\operatorname{area}(Y)} .
$$


Theorem 1.2. Let $f$ be an entire function satisfying (1.1). Then there exists $\alpha_{1}, \alpha_{2}, \eta>0$ such that if $q, \lambda \geq 0$, then $\operatorname{dens}\left(T\left(f, \alpha_{1}, \alpha_{2}, q, \lambda\right), A(R)\right)>\eta$ for sufficiently large $R$.

The proof of Theorem 1.2 is largely based on ideas of Miles and Rossi [14]; cf. the remark at the end of section 3.2 .

For an introduction to the dynamics of transcencental entire functions we refer to [4]. Results on the Hausdorff dimension of Julia sets of entire functions are surveyed in [19].

Acknowledgement 1. We thank Phil Rippon and Gwyneth Stallard for drawing our attention to Jian-Hua Zheng's paper [23].

\section{The Regularity CONDition}

2.1. Discussion of the regularity condition. We note that (1.1) implies that if $r \geq r_{0}$ and if the integer $n$ is chosen such that $C^{n} r_{0} \leq r<C^{n+1} r_{0}$, then

$$
\log M(r, f) \leq \log M\left(C^{n+1} r_{0}, f\right) \leq B^{n+1} \log M\left(r_{0}, f\right) .
$$

Since $n \leq\left(\log \left(r / r_{0}\right)\right) /(\log C)$ this implies that

$$
\log \log M(r, f) \leq n \log B+O(1) \leq \frac{\log B}{\log C} \log r+O(1)
$$

as $r \rightarrow \infty$. Hence the order $\rho(f)$ of $f$ satisfies $\rho(f) \leq(\log B) /(\log C)<\infty$. Similarly, the lower order

$$
\lambda(f)=\liminf _{r \rightarrow \infty} \frac{\log \log M(r, f)}{\log r},
$$

satisfies $\lambda(f) \geq(\log A) /(\log C)>0$.

We recall that the upper logarithmic density $\overline{\log \text { dens }} E$ of a (measurable) subset $E$ of $[1, \infty)$ is defined by.

$$
\overline{\log \operatorname{dens}} E=\limsup _{r \rightarrow \infty} \frac{1}{\log r} \int_{E \cap[1, r]} \frac{d t}{t} .
$$

It is well-known [11, Lemma 4] that if $f$ is an entire function of finite order $\rho(f)$, then the set $E$ where the right inequality of (1.1) does not hold satisfies

$$
\overline{\log \operatorname{dens}} E \leq \frac{\rho(f) \log C}{\log B}
$$

We see that $E$ is a "small" set if $B$ is large, and thus for functions $f$ of finite order (1.1) can be interpreted as a regularity condition for the growth of $f$.

2.2. Consequences of the regularity condition. It follows from (1.1) that

$$
A^{n} \log M(r, f) \leq \log M\left(C^{n} r, f\right) \leq B^{n} \log M(r, f)
$$

for $n \in \mathbb{N}$. We may thus assume without loss of generality that the constants $A, B, C$ are larger than any preassigned number. Denote by $T(r, f)$ the Nevanlinna characteristic of $f$. Using the inequality [9, 10]

$$
T(r, f) \leq \log ^{+} M(r, f) \leq \frac{R+r}{R-r} T(R, f)
$$

we see that there exists constants $A_{T}, B_{T}, C_{T}>1$ such that

$$
A_{T} T(r, f) \leq T\left(C_{T} r, f\right) \leq B_{T} T(r, f)
$$


for large $r$. For $a \in \mathbb{C}$ we denote by $n(r, a)$ the number of $a$-points of $f$ in the closed disk of radius $r$ around 0 and put

$$
N(r, a)=\int_{0}^{r} \frac{n(t, a)-n(0, a)}{t} d t+n(0, a) \log r .
$$

Denote by $E_{V}(f)$ the set of Valiron deficiencies of $f$; that is, the set of all $a$ for which

$$
\liminf _{r \rightarrow \infty} \frac{N(r, a)}{T(r, f)}<1
$$

It is well-known [9, p. 116] that area $E_{V}(f)=0$. For $a \in \mathbb{C} \backslash E_{V}(f)$ we have

$$
N(r, a) \sim T(r, f)
$$

as $r \rightarrow \infty$. Thus there exists constants $A_{N}, B_{N}, C_{N}>1$ such that if $a \in \mathbb{C} \backslash E_{V}(f)$, then

$$
A_{N} N(r, a) \leq N\left(C_{N} r, a\right) \leq B_{N} N(r, a)
$$

for sufficiently large $r$, say $r \geq r(a)$.

We note that if $M>1$, then

$$
n(r, a)=\frac{1}{\log M} \int_{r}^{M r} \frac{n(r, a)}{t} d t \leq \frac{1}{\log M} \int_{r}^{M r} \frac{n(t, a)}{t} d t \leq \frac{1}{\log M} N(M r, a)
$$

and

$$
n(M r, a) \geq \frac{1}{\log M} \int_{r}^{M r} \frac{n(t, a)}{t} d t \geq \frac{1}{\log M}(N(M r, a)-N(r, a)) \geq \frac{A_{N}-1}{\log M} N(r, a)
$$

for large $r$. With $M=C_{N}$ we see that if $a \notin E_{V}(f)$, then

$$
n\left(C_{N} r, a\right) \leq \frac{1}{\log C_{N}} N\left(C_{N}^{2} r, a\right) \leq \frac{B_{N}^{3}}{\log C_{N}} N\left(C_{N}^{-1} r, a\right) \leq \frac{B_{N}^{3}}{A_{N}-1} n(r, a)
$$

for large $r$. We obtain

$$
n\left(C_{N}^{n}, a\right) \leq\left(\frac{B_{N}^{3}}{A_{N}-1}\right)^{n} n(r, a)
$$

and choosing $n$ such that $C_{N}^{n} \geq 2$ we obtain

$$
n(2 r, a) \leq K n(r, a)
$$

with a constant $K$ for large $r$. We conclude that

$$
\log M(r, f) \leq 3 T(2 r, f) \leq 4 N(2 r, a) \leq \frac{4 \log 2}{A_{N}-1} n(4 r, a) \leq \frac{4 K^{2} \log 2}{A_{N}-1} n(r, a)
$$

for $a \notin E_{V}(f)$ and large $r$.

\section{Proof of Theorem 1.1}

3.1. An upper bound for the logarithmic derivative. In this section we consider the set

$$
U_{\tau}(f)=\left\{z \in \mathbb{C}:\left|\frac{z f^{\prime}(z)}{f(z)}\right| \leq \tau \log M(|z|, f)\right\} .
$$

We shall only need that the right inequality of (1.1) is satisfied; that is,

$$
\log M(C r, f) \leq B \log M(r, f)
$$


for large $r$. As before we deduce that

$$
\log M(2 r, f) \leq L \log M(r, f)
$$

for a constant $L$ and large $r$. Since $N(r, a) \leq T(r, f)+O(1) \leq \log M(r, f)+O(1)$ by Nevanlinna's first fundamental theorem, this implies that

$$
n(r, a) \leq \frac{1}{\log 2} N(2 r, a) \leq \frac{1}{\log 2} \log M(2 r, f)+O(1) \leq \frac{L}{\log 2} \log M(r, f)+O(1)
$$

for all $a \in \mathbb{C}$, provided $r$ is sufficiently large.

Lemma 3.1. Let $f$ be an entire satisfying (3.1). Then for each $\varepsilon>0$ there exists $\tau>0$ such that $\operatorname{dens}\left(U_{\tau}(f), A(R)\right) \geq 1-\varepsilon$ for all large $R$.

To prove this result, we shall need the following result due to Fuchs and Macintyre [8]. Here and in the following we denote by $D(a, r)$ the open disk of radius $r$ around a point $a$.

Lemma 3.2. Let $z_{1}, z_{2}, \ldots, z_{m} \in \mathbb{C}$ and let $H>0$. Then there exists $l \in\{1,2, \ldots, m\}$, $c_{1}, c_{2}, \ldots, c_{l} \in \mathbb{C}$ and $r_{1}, r_{2}, \ldots, r_{l}>0$ satisfying

$$
\sum_{k=1}^{l} r_{k}^{2} \leq 4 H^{2}
$$

such that

$$
\sum_{k=1}^{m} \frac{1}{\left|z-z_{k}\right|} \leq \frac{2 m}{H} \quad \text { for } z \in \mathbb{C}, z \notin \bigcup_{k=1}^{l} D\left(c_{k}, r_{k}\right)
$$

Proof of Lemma 3.1. For $s>|z|$ we have [9, p. 88]

$$
\left|\frac{f^{\prime}(z)}{f(z)}\right| \leq \frac{4 s}{(s-|z|)^{2}} T(s, f)+\sum_{\left|z_{j}\right| \leq s} \frac{2}{\left|z-z_{j}\right|},
$$

where $\left(z_{j}\right)$ is the sequence of zeros of $f$. (As in [9] we have assumed here that $f(0)=1$, but we may do so without loss of generality.) Now we choose $s=4 R$ so that

$$
\frac{4 s}{(s-|z|)^{2}} \leq \frac{16 R}{(4 R-2 R)^{2}}=\frac{4}{R}
$$

for $z \in A(R)$. Hence

$$
\frac{4 s}{(s-|z|)^{2}} T(s, f) \leq \frac{4 T(4 R, f)}{R} \leq \frac{4 \log M(4 R, f)}{R} \leq \frac{4 L^{2} \log M(R, f)}{R} \leq 8 L^{2} \frac{\log M(|z|, f)}{|z|}
$$

for $z \in A(R)$. To estimate the sum on the right hand side of (3.3) we use Lemma 3.2 with $H=\frac{1}{2} \sqrt{3 \varepsilon} R$ and $m=n(s, 0)$. With the notation of this lemma we have

$$
\operatorname{area}\left(\bigcup_{k=1}^{l} D\left(c_{k}, r_{k}\right)\right)=\pi \sum_{k=1}^{l} r_{k}^{2} \leq 4 \pi H^{2}=3 \varepsilon \pi R^{2}=\varepsilon \text { area } A(R)
$$

and if $z \notin \bigcup_{k=1}^{l} D\left(c_{k}, r_{k}\right)$, then

$$
\sum_{\left|z_{j}\right|<s} \frac{2}{\left|z-z_{j}\right|} \leq \frac{4 m}{H}=\frac{8}{\sqrt{3 \varepsilon}} \frac{n(4 R, 0)}{R} .
$$


Using (3.2) we see that

$$
\sum_{\left|z_{j}\right|<s} \frac{2}{\left|z-z_{j}\right|} \leq \frac{16}{\sqrt{3 \varepsilon}} \frac{L \log M(4 R, f)+O(1)}{|z|} \leq \frac{17 L^{3}}{\sqrt{3 \varepsilon}(\log 2)^{2}} \frac{\log M(|z|, f)}{|z|},
$$

provided $R$ is sufficiently large. The conclusion follows with

$$
\tau=8 L^{2}+\frac{17 L^{3}}{\sqrt{3 \varepsilon}(\log 2)^{2}} .
$$

The proof actually yields the following result.

Lemma 3.3. Let $f$ be an entire satisfying (3.1). Then for each $\varepsilon>0$ there exists $\tau>0$ such that if $R$ is sufficiently large, then there exist $l \leq n(4 R, 0)$ and $c_{1}, \ldots, c_{l} \in D(0,4 R)$ and $r_{1}, \ldots, r_{l}>0$ such that

$$
A(R) \backslash U_{\tau}(f) \subset \bigcup_{k=1}^{l} D\left(c_{k}, r_{k}\right) \quad \text { and } \quad \sum_{k=1}^{l} r_{k}^{2} \leq \varepsilon R^{2}
$$

3.2. A lower bound for the logarithmic derivative. The results of this subsection are minor modifications of results of Miles and Rossi [14]. The differences between their results and the results below are explained at the end of this subsection.

Let $f$ be an entire function of finite order $\rho(f)$ and denote by $n(r)$ the number of zeros of $f$ in the disk of radius $r$ around 0 .

Lemma 3.4. Suppose that there exists $r_{0}>0$ and $K>1$ such that

$$
n(2 r) \leq K n(r) \quad \text { for } r \geq r_{0} .
$$

For $\mu>0$ let $F_{\mu}$ be the set of all $r \geq r_{0}$ for which

$$
n(t) \leq\left(\frac{t}{r}\right)^{\mu} n(r) \quad \text { for } t \geq r
$$

while

$$
n(t) \geq\left(\frac{t}{r}\right)^{\mu} n(r) \quad \text { for } r_{0} \leq t \leq r .
$$

Then, given $\delta>0$, there exists $\mu>0$ such that

$$
\operatorname{meas}\left(F_{\mu} \cap[R, 2 R]\right) \geq(1-\delta) R
$$

for all $R \geq 2 r_{0}$.

Proof. It follows from (3.4) that if $t \geq 2 r$ and if $m \in \mathbb{N}$ is chosen such that $2^{m} r \leq t<$ $2^{m+1} r$, then

$$
n(t) \leq K^{m+1} n\left(2^{-m-1} t\right) \leq K^{m+1} n(r) \leq K^{2 m} n(r)=\exp (2 m \log K) n(r) .
$$

We also have $m \leq(\log (t / r)) /(\log 2)$ and thus

$$
n(t) \leq \exp \left(2 \log \left(\frac{t}{r}\right) \frac{\log K}{\log 2}\right) n(r)=\left(\frac{t}{r}\right)^{\frac{2 \log K}{\log 2}} n(r)
$$


We see that if

$$
\mu \geq \frac{2 \log K}{\log 2}
$$

then

$$
n(t) \leq\left(\frac{t}{r}\right)^{\mu} n(r) \quad \text { for } t \geq 2 r
$$

so that condition (3.5) is satisfied as soon as

$$
n(t) \leq\left(\frac{t}{r}\right)^{\mu} n(r) \quad \text { for } r \leq t \leq 2 r .
$$

Let now $R \geq 2 r_{0}$ and let $E_{1}$ be the set of all $r \in[R, 2 R]$ where (3.5) does not hold and let $E_{2}$ be the set of all $r \in[R, 2 R]$ where (3.6) does not hold. We shall show that

$$
\text { meas } E_{1} \leq \frac{1}{2} \delta R \text { and meas } E_{2} \leq \frac{1}{2} \delta R
$$

if $\mu$ is chosen large enough. The conclusion then follows.

To prove the claim about $E_{1}$ we may assume that $E_{1} \neq \emptyset$ and choose

$$
s_{1} \in E_{1} \cap\left[\inf E_{1}, \inf E_{1}+\frac{1}{8} \delta R\right]
$$

Then there exists $t_{1}>s_{1}$ with

$$
n\left(t_{1}\right)>\left(\frac{t_{1}}{s_{1}}\right)^{\mu} n\left(s_{1}\right)
$$

and because of (3.8) we have $t_{1} \leq 2 s_{1} \leq 4 R$. Inductively we define

$$
s_{k} \in E_{1} \cap\left[\inf \left(E_{1} \cap\left[t_{k-1}, 2 R\right]\right), \inf \left(E_{1} \cap\left[t_{k-1}, 2 R\right]\right)+2^{-k-2} \delta R\right]
$$

and choose $t_{k} \in\left(s_{k}, 2 s_{k}\right]$ with

$$
n\left(t_{k}\right)>\left(\frac{t_{k}}{s_{k}}\right)^{\mu} n\left(s_{k}\right)
$$

as long as $E_{1} \cap\left[t_{k-1}, 2 R\right] \neq \emptyset$. However, noting that $n\left(t_{k}\right)>n\left(s_{k}\right) \geq n\left(t_{k-1}\right)$ and $n\left(t_{k}\right) \leq n\left(2 s_{k}\right) \leq n(4 R)$ we see that the process terminates so that there exists $N \in \mathbb{N}$ with $E_{1} \cap\left[t_{N}, 2 R\right]=\emptyset$ and

$$
E_{1} \subset \bigcup_{k=1}^{N}\left[s_{k}-2^{-k-2} \delta R, t_{k}\right] .
$$

Since $s_{k} \geq t_{k-1}$ it follows from $(\underline{3.9})$ that

$$
n\left(t_{k}\right)>\left(\frac{t_{k}}{s_{k}}\right)^{\mu} n\left(t_{k-1}\right)
$$

and hence that

$$
n(4 R) \geq n\left(t_{N}\right)>n(R) \prod_{k=1}^{N}\left(\frac{t_{k}}{s_{k}}\right)^{\mu} .
$$

Since $n(4 R) \leq K^{2} n(r)$ by (3.4) this yields

$$
\prod_{k=1}^{N}\left(\frac{t_{k}}{s_{k}}\right)^{\mu} \leq K^{2}
$$


and thus

$$
\mu \sum_{k=1}^{N} \log \frac{t_{k}}{s_{k}} \leq 2 \log K
$$

Since $0 \leq t_{k}-s_{k} \leq s_{k} \leq 2 R$ and since $\log (1+x) \geq x \log 2$ for $0 \leq x \leq 1$ we have

$$
\log \frac{t_{k}}{s_{k}}=\log \left(1+\frac{t_{k}-s_{k}}{s_{k}}\right) \geq \frac{t_{k}-s_{k}}{s_{k}} \log 2 \geq\left(t_{k}-s_{k}\right) \frac{\log 2}{2 R}
$$

and thus

$$
\sum_{k=1}^{N}\left(t_{k}-s_{k}\right) \leq \frac{2 R}{\log 2} \sum_{k=1}^{N} \log \frac{t_{k}}{s_{k}} \leq \frac{4 R \log K}{\mu \log 2} .
$$

Choosing $\mu>(16 \log K) /(\delta \log 2)$ we obtain

$$
\sum_{k=1}^{N}\left(t_{k}-s_{k}\right) \leq \frac{1}{4} \delta R
$$

and thus

$$
\text { meas } E_{1} \leq \sum_{k=1}^{N}\left(t_{k}-s_{k}+2^{-k-2} \delta R\right) \leq \frac{1}{2} \delta R \text {. }
$$

The estimate for $E_{2}$ is similar. Here we choose

$$
s_{1} \in E_{2} \cap\left[\sup E_{2}-\frac{1}{8} \delta R, \sup E_{2}\right]
$$

and $r_{1} \in\left[r_{0}, s_{1}\right)$ with

$$
n\left(r_{1}\right)<\left(\frac{r_{1}}{s_{1}}\right)^{\mu} n\left(s_{1}\right) .
$$

It follows from (3.7) that $r_{1} \in\left[\frac{1}{2} s_{1}, s_{1}\right)$. Inductively we choose

$$
s_{k} \in E_{2} \cap\left[\sup \left(E_{2} \cap\left[R, r_{k-1}\right]\right)-2^{-k-2} \delta R, \sup \left(E_{2} \cap\left[R, r_{k-1}\right]\right)\right]
$$

and $r_{k} \in\left[\frac{1}{2} s_{k}, s_{k}\right)$ with

$$
n\left(r_{k}\right)<\left(\frac{r_{k}}{s_{k}}\right)^{\mu} n\left(s_{k}\right) .
$$

Again the process stops and there exists $N \in \mathbb{N}$ with

$$
E_{2} \subset \bigcup_{k=1}^{N}\left[r_{k}, s_{k}+2^{-k-2} \delta R\right]
$$

and

$$
n\left(\frac{1}{2} R\right) \leq n\left(r_{N}\right) \leq n\left(s_{1}\right) \prod_{k=1}^{N}\left(\frac{r_{k}}{s_{k}}\right)^{\mu} \leq K^{2} n\left(\frac{1}{2} R\right) \prod_{k=1}^{N}\left(\frac{r_{k}}{s_{k}}\right)^{\mu} .
$$

Thus

$$
\mu \sum_{k=1}^{N} \log \frac{s_{k}}{r_{k}} \leq 2 \log K
$$

and as before this yields

$$
\text { meas } E_{2} \leq \sum_{k=1}^{N}\left(s_{k}-r_{k}+2^{-k-2} \delta R\right) \leq \frac{1}{2} \delta
$$


We note that follows from (3.5) and (3.6) that $n(t)$ is continuous at $t=r$, meaning that there is no zero of $f$ on the circle of radius $r$ around 0 . We also note that (3.5) and (3.6) remain valid if $\mu$ is replaced by a larger number.

We shall assume that $f(0) \neq 0$ and denote by $\left(z_{j}\right)$ the sequence of zeros of $f$, ordered such that $\left|z_{1}\right| \leq\left|z_{2}\right| \leq \ldots$. Replacing $K$ by a larger number if necessary, we may assume that (3.4) holds with $r_{0}=\left|z_{1}\right|$.

For $0<\beta<1$ and $r \geq 0$ we put

$$
U(r)=\left\{\theta \in[0,2 \pi]:\left|\frac{f^{\prime}\left(r e^{i \theta}\right)}{f\left(r e^{i \theta}\right)}\right| \geq \beta \frac{n(r)}{r}\right\} .
$$

Lemma 3.5. If (3.5) and (3.6) hold for some $\mu \geq \max \{\rho(f), 1\}$, then

$$
\text { meas } U(r) \geq \frac{2 \pi(1-\beta)^{2}}{(\beta+3 \pi \mu)^{2}}
$$

provided $r$ is sufficiently large.

Proof. We put $q=[\mu+1]$ and write

$$
f(z)=e^{P(z)} \prod_{j=1}^{\infty} E\left(\frac{z}{z_{j}}, q\right)
$$

where $P(z)=\sum_{m=0}^{q} a_{m} z^{m}$ is a polynomial of degree at most $q$ and where $E(\cdot, q)$ denotes the Weierstraß primary factor. Note that $q$ will in general be much larger than $\rho(f)$ so that the above form of $f$ is not the usual Hadamard factorization. We put

$$
L(\theta)=r e^{i \theta} \frac{f^{\prime}\left(r e^{i \theta}\right)}{f\left(r e^{i \theta}\right)} .
$$

Then

$$
L(\theta)=r e^{i \theta} P^{\prime}\left(r e^{i \theta}\right)+\sum_{m=-\infty}^{\infty} b_{m}(r) e^{i m \theta}
$$

where [21, p. 350]

$$
b_{m}(r)=-\sum_{\left|z_{j}\right|>r}\left(\frac{z_{j}}{r}\right)^{-m} \quad \text { for } m>q
$$

while

$$
b_{m}(r)=\sum_{\left|z_{j}\right|<r}\left(\frac{z_{j}}{r}\right)^{-m} \quad \text { for } m<0 .
$$


For $m>q$ we deduce from (3.5) that

$$
\begin{aligned}
\left|b_{m}(r)\right| & \leq \sum_{\left|z_{j}\right|>r}\left(\frac{\left|z_{j}\right|}{r}\right)^{-m} \\
& =\int_{r}^{\infty}\left(\frac{t}{r}\right)^{-m} d n(t) \\
& =-n(r)+m \int_{r}^{\infty}\left(\frac{t}{r}\right)^{-m} n(t) \frac{d t}{t} \\
& \leq-n(r)+m n(r) \int_{r}^{\infty}\left(\frac{t}{r}\right)^{\mu-m} \frac{d t}{t} \\
& =n(r) \frac{\mu}{m-\mu} .
\end{aligned}
$$

Thus

$$
\sum_{m>q}\left|b_{m}(r)\right|^{2} \leq \frac{\pi^{2}}{6} \mu^{2} n(r)^{2}
$$

Similarly we find for $m<0$ that

$$
\begin{aligned}
\left|b_{m}(r)\right| & \leq \int_{r_{0} / 2}^{r}\left(\frac{t}{r}\right)^{-m} d n(t) \\
& =n(r)+m \int_{r_{0}}^{r}\left(\frac{t}{r}\right)^{-m} n(t) \frac{d t}{t} \\
& \leq n(r)+m n(r) \int_{r_{0}}^{r}\left(\frac{t}{r}\right)^{\mu-m} \frac{d t}{t} \\
& =n(r)\left(\frac{\mu}{\mu-m}+m\left(\frac{r_{0}}{r}\right)^{\mu-m}\right) .
\end{aligned}
$$

For large $r$ we thus have

$$
\left|b_{m}(r)\right| \leq \frac{2 \mu}{\mu-m} n(r)
$$

for all $m<0$ and this yields

$$
\sum_{m<0}\left|b_{m}(r)\right|^{2} \leq \frac{2 \pi^{2}}{3} \mu^{2} n(r)^{2}
$$

With

$$
g(\theta)=r e^{i \theta} P^{\prime}\left(r e^{i \theta}\right)+\sum_{m=0}^{q} b_{m}(r) e^{i m \theta}=\sum_{m=0}^{q}\left(m a_{m} r^{m}+b_{m}(r)\right) e^{i m \theta}
$$

we thus have

$$
L(\theta)=g(\theta)+s(\theta)
$$

where

$$
\|s\|_{2}^{2}=\frac{1}{2 \pi} \int_{0}^{2 \pi}|s(\theta)|^{2} d \theta=\sum_{m<0}\left|b_{m}(r)\right|^{2}+\sum_{m>q}\left|b_{m}(r)\right|^{2} \leq \pi^{2} \mu^{2} n(r)^{2}
$$


so that

$$
\|s\|_{2} \leq \pi \mu n(r) .
$$

In order to estimate $\|g\|_{2}$ we write

$$
|g(\theta)|^{2}=\sum_{m=-q}^{q} h_{m}(r) e^{i m \theta}
$$

and note that $h_{-m}(r)=\overline{h_{m}(r)}$ and

$$
\left|h_{m}(r)\right|=\left.\left.\left|\frac{1}{2 \pi} \int_{0}^{2 \pi}\right| g(\theta)\right|^{2} e^{-i m \theta} d \theta\left|\leq\|g\|_{2}^{2}=\frac{1}{2 \pi} \int_{0}^{2 \pi}\right| g(\theta)\right|^{2} d \theta=h_{0}(r)
$$

for all $m$. Let now $V(r)=[0,2 \pi] \backslash U(r)$ so that

$$
|L(\theta)|<\beta n(r) \quad \text { for } \theta \in V(r) .
$$

Since

$$
0=\int_{0}^{2 \pi} h_{m}(r) e^{i m \theta} d \theta=\int_{U(r)} h_{m}(r) e^{i m \theta} d \theta+\int_{V(r)} h_{m}(r) e^{i m \theta} d \theta
$$

for $m \neq 0$ we deduce from (3.13) that

$$
\begin{aligned}
\int_{V(r)}|g(\theta)|^{2} d \theta & =\int_{V(r)} h_{0}(r) d \theta-\sum_{1 \leq|m| \leq q} \int_{U(r)} h_{m}(r) e^{i m \theta} d \theta \\
& \geq h_{0}(r) \text { meas } V(r)-\sum_{1 \leq|m| \leq q}\left|h_{m}(r)\right| \text { meas } U(r) \\
& \geq h_{0}(r) \text { meas } V(r)-2 q h_{0}(r) \text { meas } U(r) \\
& =h_{0}(r)(\text { meas } V(r)-2 q \text { meas } U(r)) .
\end{aligned}
$$

If meas $U(r) \geq \pi /(2 q+1)$, then (3.10) follows since $q \leq \mu+1$ so that $2 q+1 \leq 2 \mu+3 \leq 5 \mu$ and this yields

$$
\frac{\pi}{2 q+1} \geq \frac{2 \pi(1-\beta)^{2}}{(\beta+3 \pi \mu)^{2}}
$$

We may thus assume that meas $U(r)<\pi /(2 q+1)$ so that meas $V(r)>2 \pi-\pi /(2 q+1)$. We deduce from (3.13) and (3.15) that

$$
\int_{V(r)}|g(\theta)|^{2} d \theta \geq\left(2 \pi-\frac{\pi}{2 q+1}-2 q \frac{\pi}{2 q+1}\right) h_{0}(r)=\pi\|g\|_{2}^{2} .
$$

Using (3.11), (3.12) and (3.14) we find that

$$
\begin{aligned}
\frac{1}{\sqrt{2}}\|g\|_{2} & \leq\left(\frac{1}{2 \pi} \int_{V(r)}|g(\theta)|^{2} d \theta\right)^{1 / 2} \\
& \leq\left(\frac{1}{2 \pi} \int_{V(r)}|L(\theta)|^{2} d \theta\right)^{1 / 2}+\left(\frac{1}{2 \pi} \int_{V(r)}|s(\theta)|^{2} d \theta\right)^{1 / 2} \\
& \leq \beta n(r)+\pi \mu n(r)
\end{aligned}
$$

and hence

$$
\|g\|_{2} \leq(\beta+2 \pi \mu) n(r)
$$


Combining this with (3.11) and (3.12) we conclude that

$$
\|L\|_{2} \leq\|g\|_{2}+\|s\|_{2} \leq(\beta+3 \pi \mu) n(r) .
$$

On the other hand, it follows from the argument principle that

$$
n(r)=\frac{1}{2 \pi i} \int_{|z|=r} \frac{f^{\prime}(z)}{f(z)} d z=\frac{1}{2 \pi} \int_{0}^{2 \pi} L(\theta) d \theta=\frac{1}{2 \pi} \int_{U(r)} L(\theta) d \theta+\frac{1}{2 \pi} \int_{V(r)} L(\theta) d \theta .
$$

Now the Cauchy-Schwarz inequality, (3.14) and (3.16) yield

$$
\begin{aligned}
n(r) & \leq \frac{1}{2 \pi}\left(\int_{U(r)} d \theta\right)^{1 / 2}\left(\int_{U(r)}|L(\theta)|^{2} d \theta\right)^{1 / 2}+\beta n(r) \\
& \leq \frac{1}{\sqrt{2 \pi}} \sqrt{\text { meas } U(r)}\|L\|_{2}+\beta n(r) \\
& \leq \frac{1}{\sqrt{2 \pi}} \sqrt{\text { meas } U(r)}(\beta+3 \pi \mu) n(r)+\beta n(r)
\end{aligned}
$$

Hence

$$
\text { meas } U(r) \geq \frac{2 \pi(1-\beta)^{2}}{(\beta+3 \pi \mu)^{2}}
$$

Remark. It was shown at the beginning of the proof of Lemma 3.4 that if $n(r)$ satisfies (3.4), then there exists $\rho>0$ such that

$$
n(r)=O\left(r^{\tau}\right)
$$

as $r \rightarrow \infty$. (In fact, the argument shows that we can take $\tau=(2 \log K) /(\log 2)$, and a slightly more careful estimate will give $\tau=(\log K) /(\log 2)$.)

Miles and Rossi [14] show that if $n(r)$ satisfies (3.17), then (3.5) and (3.6) hold on a set of logarithmic density $1-\delta$ if $\mu$ is sufficiently large. They then use this to show that (3.10) holds on a set of logarithmic density $1-\delta$.

For our applications, however, a set of positive logarithmic density is not sufficient. Therefore we introduced the additional hypothesis (3.4). Lemma 3.4 says that with this additional hypothesis (3.10) holds on a set of density $1-\delta$.

The proof of Lemma 3.5, which says that (3.5) and (3.6) imply (3.10), is essentially the same as that of Miles and Rossi [14] and it is included here only for completeness.

We also note that (3.17) implies that there exists a constant $K$ such that (3.4) holds on a set of positive density. In fact, by taking $K$ large this density can be taken arbitrarily close to 1.

3.3. Completion of the proof of Theorem 1.2. Let $a \in \mathbb{C} \backslash E_{V}(f)$. By (2.2) we can apply Lemma 3.4 to $f-a$. With

$$
\gamma=\frac{\beta\left(A_{N}-1\right)}{4 K^{2} \log 2} \quad \text { and } \quad c=\frac{1-\delta}{2} \frac{(1-\beta)^{2}}{(\beta+3 \pi \mu)^{2}}
$$

and with

$$
V_{\gamma}(a)=\left\{z \in \mathbb{C}:\left|\frac{z f^{\prime}(z)}{f(z)-a}\right| \geq \gamma \log M(|z|, f)\right\}
$$


we deduce from Lemmas 3.4 and 3.5 and from (2.3) that $\operatorname{dens}\left(V_{\gamma}(a), A(R)\right) \geq c$ for large $R$. We apply Lemma 3.1 with $\varepsilon=\frac{1}{4} c$ to $f-a$ and with

$$
U_{\tau}(f-a)=\left\{z \in \mathbb{C}:\left|\frac{z f^{\prime}(z)}{f(z)-a}\right| \leq \tau \log M(|z|, f)\right\}
$$

we obtain $\operatorname{dens}\left(U_{\tau}(f-a), A(R)\right) \geq 1-\varepsilon$ if $\tau$ is sufficiently large.

We put $d=\tau / \gamma$, fix $m \geq 1 / \varepsilon$ and choose $a_{1}, \ldots, a_{m} \in D(0,2 d m) \backslash E_{V}(f)$ with $\left|a_{j}-a_{k}\right| \geq 2 d$ for $j \neq k$. For $1 \leq j \leq m$ we put

$$
C_{j}=\left\{z \in \mathbb{C}:\left|f(z)-a_{j}\right| \leq d\right\}
$$

Then the $C_{j}$ are pairwise disjoint and thus there exists $j=j(R)$ with $\operatorname{dens}\left(C_{j}, A(R)\right) \leq \varepsilon$. With

$$
\left.W\left(a_{j}\right)=\left(U_{\tau}\left(f-a_{j}\right) \cap U_{\tau}(f)\right) \cap V_{\gamma}\left(a_{j}\right)\right) \backslash C_{j}
$$

we deduce from Lemma 3.1 that $\operatorname{dens}\left(W\left(a_{j}\right), A(R)\right) \geq \frac{1}{4} c$. For $z \in W\left(a_{j}\right) \cap A(R)$ we have

and thus

$$
\gamma \log M(|z|, f) \leq\left|\frac{z f^{\prime}(z)}{f(z)-a_{j}}\right| \leq \frac{\left|z f^{\prime}(z)\right|}{d}
$$

$$
\left|z f^{\prime}(z)\right| \geq d \gamma \log M(|z|, f)=\tau \log M(|z|, f) \geq\left|\frac{z f^{\prime}(z)}{f(z)}\right| .
$$

Hence $|f(z)| \geq 1$ for $z \in W\left(a_{j}\right) \cap A(R)$. Moreover, if $|f(z)| \leq 4 d m$, then we have

$$
\left|\frac{z f^{\prime}(z)}{f(z)}\right|=\left|\frac{z f^{\prime}(z)}{f(z)-a_{j}} \frac{f(z)-a_{j}}{f(z)}\right| \geq \frac{1}{4 m}\left|\frac{z f^{\prime}(z)}{f(z)-a_{j}}\right| \geq \frac{\gamma}{4 m} \log M(|z|, f)
$$

and if $|f(z)| \geq 4 d m$, then $|f(z)| \geq 2\left|a_{j}\right|$ and thus

$$
\left|\frac{z f^{\prime}(z)}{f(z)}\right|=\left|\frac{z f^{\prime}(z)}{f(z)-a_{j}}\left(1-\frac{a_{j}}{f(z)}\right)\right| \geq \frac{1}{2}\left|\frac{z f^{\prime}(z)}{f(z)-a_{j}}\right| \geq \frac{\gamma}{2} \log M(|z|, f) .
$$

Summarizing the above estimates we obtain with $\sigma=\gamma /(4 m)$ that if $z \in W\left(a_{j}\right) \cap A(R)$, then

$$
\sigma \log M(|z|, f) \leq\left|\frac{z f^{\prime}(z)}{f(z)}\right| \leq \tau \log M(|z|, f) \quad \text { and } \quad|f(z)| \geq 1 .
$$

Noting that $|z|^{0}=1$ we have thus proved that if $f$ satisfies (2.1), then

$$
\operatorname{dens}(T(f, \sigma, \tau, 0,0), A(R)) \geq \frac{1}{4} c
$$

for all large $R$. In other words, we have proved the special case $q=\lambda=0$ of our theorem. We may apply this result to

$$
g(z)=\frac{f(z)-a}{P(z)}
$$

where $a$ is chosen such that $f$ has infinitely many $a$-points and where $P$ is a polynomial of degree greater than $q$ whose zeros are $a$-points of $f$. In fact, we have

$$
\log M(r, g)=\log M(r, f)+O(\log r)=(1+o(1)) \log M(r, f)
$$

as $r \rightarrow \infty$ so that (2.1) holds with $f$ replaced by $g$ if the constants $A$ and $C$ are slightly adjusted. Hence dens $\left(T\left(g, \sigma^{*}, \tau^{*}, 0,0\right), A(R)\right) \geq \eta$ if $0<\sigma^{*}<\sigma, \tau^{*}>\tau$ and $0<\eta<\frac{1}{4} c$, provided $R$ is large enough. 
Now

$$
\frac{z g^{\prime}(z)}{g(z)}=\frac{z f^{\prime}(z)}{f(z)-a}-\frac{z P^{\prime}(z)}{P(z)}=\frac{z f^{\prime}(z)}{f(z)} \frac{f(z)}{f(z)-a}-\frac{z P^{\prime}(z)}{P(z)} .
$$

For $z \in T\left(g, \sigma^{*}, \tau^{*}, 0,0\right)$ we have $|g(z)| \geq 1$ and thus $|f(z)|=|P(z) g(z)+a| \geq|z|^{q}$, provided $|z|$ is sufficiently large. Thus

$$
\frac{f(z)}{f(z)-a} \rightarrow 1
$$

as $|z| \rightarrow \infty, z \in T\left(g, \sigma^{*}, \tau^{*}, 0,0\right)$. Since

$$
\frac{z P^{\prime}(z)}{P(z)} \rightarrow \operatorname{deg} P
$$

as $|z| \rightarrow \infty$ we conclude that if $0<\alpha_{1}<\sigma^{*}$ and $\alpha_{2}>\tau^{*}$, then

$$
T\left(g, \sigma^{*}, \tau^{*}, 0,0\right) \backslash D(0, S) \subset T\left(f, \alpha_{1}, \alpha_{2}, q, 0\right)
$$

for large $S$ and hence dens $\left(T\left(f, \alpha_{1}, \alpha_{2}, q, 0\right), A(R)\right) \geq \eta$ for large $R$. This is the special case $\lambda=0$ of our theorem.

In order to obtain this result for general $\lambda$, we apply Lemma 3.3. We note that if $z \in A(R)$, then $|z| / \log M(|z|, f) \leq 2 R / \log M(R, f)$. This implies that if $c_{1}, \ldots, c_{l}$ and $r_{1}, \ldots, r_{l}$ are as in Lemma 3.3 and if $z \in A(R) \backslash \bigcup_{k=1}^{l} D\left(c_{k}, r_{k}+2 \lambda R / \log M(R, f)\right)$, then $D(z, \lambda|z| / \log M(|z|, f)) \cap \bigcup_{k=1}^{l} D\left(c_{k}, r_{k}\right)=\emptyset$. Thus

$$
\left|\frac{\zeta f^{\prime}(\zeta)}{f(\zeta)}\right| \leq \tau \frac{\log M(|\zeta|, f)}{|\zeta|} \quad \text { for } \quad z \in D\left(z, \lambda \frac{|z|}{\log M(|z|, f)}\right)
$$

provided

$$
z \in A(R) \backslash \bigcup_{k=1}^{l} D\left(c_{k}, r_{k}+\frac{2 \lambda R}{\log M(R, f)}\right) .
$$

Using $(a+b)^{2} \leq 2\left(a^{2}+b^{2}\right)$ and (3.1) and (3.2) we see that

$$
\begin{aligned}
\sum_{k=1}^{l}\left(r_{k}+\frac{2 \lambda R}{\log M(R, f)}\right)^{2} & \leq 2 \sum_{k=1}^{l} r_{k}^{2}+\frac{8 \lambda^{2} R^{2}}{(\log M(R, f))^{2}} n(4 R, 0) \\
& \leq 2 \varepsilon R^{2}+\frac{8 \lambda^{2} L^{3} R^{2}}{(\log 2) \log M(R, f)} \\
& \leq 3 \varepsilon R^{2} .
\end{aligned}
$$

We see that the density of the set of all $z \in A(R)$ for which (1.6) fails can be made arbitrarily small by choosing $\alpha_{2}$ large. The conclusion follows.

\section{Auxiliary results for the proof of Theorem 1.1}

The following lemma can be proved by a simple compactness argument.

Lemma 4.1. Let $\Omega$ be a domain let $Q$ be a compact subset of $\Omega$. Then there exists a positive constant $C$ such that if $f$ is univalent in $\Omega$ and $z, \zeta \in Q$, then $\left|f^{\prime}(\zeta)\right| \leq C\left|f^{\prime}(z)\right|$.

In principle this lemma would be sufficient for our purposes, but we note that the classical Koebe distortion theorem gives explicit estimates in the case where $\Omega$ is a disk. 
Lemma 4.2. Let $f$ be univalent in $D(a, r)$ and let $z \in \overline{D(a, \rho r)}$, where $0<\rho<1$. Then

$$
\frac{1-\rho}{(1+\rho)^{3}}\left|f^{\prime}(a)\right| \leq\left|f^{\prime}(z)\right| \leq \frac{1+\rho}{(1-\rho)^{3}}\left|f^{\prime}(a)\right| \text {. }
$$

We shall use the following version of the Ahlfors islands theorem; cf. [10, Theorem 6.2].

Lemma 4.3. Let $D_{1}, D_{2}, D_{3}$ be Jordan domains with pairwise disjoint closures. Then there exists $\mu>0$ with the following property: if $a \in \mathbb{C}, r>0$ and $f: D(a, r) \rightarrow \mathbb{C}$ is a holomorphic function satisfying

$$
\frac{\left|f^{\prime}(a)\right|}{1+|f(a)|^{2}} \geq \frac{\mu}{r}
$$

then $D(a, r)$ has a subdomain which is mapped bijectively onto one of the domains $D_{\nu}$.

To estimate the Hausdorff dimension we will use a result of McMullen [13]. In order to state it, consider for $l \in \mathbb{N}$ a collection $\mathcal{E}_{l}$ of disjoint compact subsets of $\mathbb{R}^{n}$ such that the following two conditions are satisfied:

(a) every element of $\mathcal{E}_{l+1}$ is contained in a unique element of $\mathcal{E}_{l}$;

(b) every element of $\mathcal{E}_{l}$ contains at least one element of $\mathcal{E}_{l+1}$.

Denote by $E_{l}$ the union of all elements of $\mathcal{E}_{l}$ and put $E=\bigcap_{l=1}^{\infty} E_{l}$. Suppose that $\left(\Delta_{l}\right)$ and $\left(d_{l}\right)$ are sequences of positive real numbers such that if $F \in \mathcal{E}_{l}$, then

$$
\operatorname{dens}\left(E_{l+1}, F\right) \geq \Delta_{l}
$$

and

$$
\operatorname{diam} F \leq d_{l}
$$

Then we have the following result [13, Proposition 2.2].

Lemma 4.4. Let $E, \mathcal{E}_{l}, \Delta_{l}$ and $d_{l}$ be as above. Then

$$
\limsup _{l \rightarrow \infty} \frac{\sum_{j=1}^{l}\left|\log \Delta_{j}\right|}{\left|\log d_{l}\right|} \geq n-\operatorname{dim} E .
$$

The following result is due to Zheng [23, Corollary 5].

Lemma 4.5. Let $f$ be an entire function satisfying (1.1). Then the Fatou set of $f$ has no multiply connected components.

Actually Zheng requires only that the left inequality of (1.1) holds. More precisely, he assumes that there exists $d>1$ such that $\log M(2 r, f) \geq d \log M(r, f)$ for all large $r$, but we may replace $M(2 r, f)$ by $M(C r, f)$ here if $C>1$.

\section{Proof of Theorem 1.1}

Let $f$ be an entire function satisfying (1.1) and let $\alpha_{1}, \alpha_{2}, \eta$ be as in Theorem 1.2. We apply Lemma 4.3 to the domains

$$
D_{\nu}=\{z \in \mathbb{C}:|\operatorname{Re} z|<1,|\operatorname{Im} z-8 \pi \nu|<3 \pi\}, \quad \text { for } \nu \in\{1,2,3\} .
$$

Let $\mu$ be such that the conclusion of Lemma 4.3 is satisfied for these domains and let $\lambda=2 L \mu / \alpha_{1}$ where $L$ is the constant from (3.1). We will apply Theorem 1.2 with this value of $\lambda$ and with $q=8$. 
For large $R$ we put $t=t(R)=2 \mu R /\left(\alpha_{1} \log M(R, f)\right)$. Note that (1.6) implies in particular that $f$ has no zeros in $D(z, \lambda|z| / \log M(|z|, f))$ if $z \in T\left(f, \alpha_{1}, \alpha_{2}, q, \lambda\right)$. Since

$$
\frac{\lambda|z|}{\log M(|z|, f)} \geq \frac{\lambda R}{\log M(2 R, f)} \geq \frac{\lambda R}{L \log M(R, f)}=t(R)
$$

for $z \in A(R)$ by (3.1) this implies that a branch of $\log f$ can be defined in the disk $D(z, t(R))$, provided $z \in T\left(f, \alpha_{1}, \alpha_{2}, q, \lambda\right) \cap A(R)$.

Lemma 5.1. Let $a \in T\left(f, \alpha_{1}, \alpha_{2}, q, \lambda\right) \cap A(R)$. If $R$ is sufficiently large, then $D(a, t(R))$ contains a subdomain $U$ such that $\log f$ maps $U$ bijectively onto one of the domains

$$
\begin{aligned}
\Omega_{\nu}(a) & =\log f(a)+D_{\nu} \\
& =\{z \in \mathbb{C}:|\operatorname{Re}(z-\log f(a))|<1,|\operatorname{Im}(z-\log f(a))-8 \pi \nu|<3 \pi\} .
\end{aligned}
$$

Moreover, there exist $\beta, \gamma>0$ such that if $V$ is the subset of $U$ which is mapped onto

$$
Q_{\nu}(a)=\{z \in \mathbb{C}: 0 \leq \operatorname{Re}(z-\log f(a)) \leq \log 2,|\operatorname{Im}(z-\log f(a))-8 \pi \nu| \leq 2 \pi\},
$$

then area $V \geq \beta t(R)^{2}$ and

$$
\left|\frac{f^{\prime}(z)}{f(z)}\right| \geq \frac{\gamma}{t(R)} \quad \text { for } z \in V .
$$

Proof. Let $h: D(a, t) \rightarrow \mathbb{C}, h(z)=\log f(z)-\log f(a)$. Then $h(a)=0$ and thus

$$
\frac{\left|h^{\prime}(a)\right|}{1+|h(a)|^{2}}=\left|h^{\prime}(a)\right|=\frac{\left|f^{\prime}(a)\right|}{|f(a)|} \geq \alpha_{1} \frac{\log M(|z|, f)}{|z|} \geq \frac{\alpha_{1}}{2} \frac{\log M(R, f)}{R}=\frac{\mu}{t}
$$

Lemma 4.3 implies that there exists a subdomain $U$ of $D(a, t)$ which is mapped by $h$ bijectively onto one of the three domain $D_{\nu}$ occuring in this lemma. It follows that $\log f$ maps $U$ bijectively onto one of the domains $\log f(a)+D_{\nu}$.

We have

$$
\begin{aligned}
4 \pi \log 2 & =\operatorname{area} Q_{\nu} \\
& =\operatorname{area} h(V) \\
& \leq \sup _{z \in V}\left|h^{\prime}(z)\right|^{2} \text { area } V \\
& \leq \sup _{z \in V}\left(\alpha_{2} \frac{\log M(|z|, f)}{|z|}\right)^{2} \text { area } V \\
& \leq\left(\alpha_{2} L \frac{\log M(R, f)}{R}\right)^{2} \text { area } V \\
& =\left(\frac{2 \mu \alpha_{2} L}{\alpha_{1} t}\right)^{2} \text { area } V
\end{aligned}
$$

With $\beta=\left(\pi \alpha_{1}^{2} \log 2\right) /\left(\mu \alpha_{2} L\right)^{2}$ we thus have area $V \geq \beta t^{2}$.

By Lemma 4.1, there exists a constant $C>1$ such if $\phi$ denotes the branch of the inverse of $h$ which maps $D_{\nu}$ to $U$, then $\left|\phi^{\prime}(\zeta)\right| \leq C\left|\phi^{\prime}(z)\right|$ for $z, \zeta \in Q_{\nu}$. It follows that $\left|h^{\prime}(\zeta)\right| \leq C\left|h^{\prime}(z)\right|$ for $z, \zeta \in V$. Thus

$$
4 \pi \log 2 \leq \sup _{z \in V}\left|h^{\prime}(z)\right|^{2} \text { area } V \leq C^{2} \inf _{z \in V}\left|h^{\prime}(z)\right|^{2} \text { area } V \leq C^{2} \pi t^{2} \inf _{z \in V}\left|h^{\prime}(z)\right|^{2}
$$


so that

$$
\inf _{z \in V}\left|h^{\prime}(z)\right| \geq \frac{2 \sqrt{\log 2}}{C t} .
$$

Since $h^{\prime}=f^{\prime} / f$ we see that (5.1) follows with $\gamma=2 \sqrt{\log 2} / C$.

If $U$ is as in Lemma 5.1, then $f$ maps $U$ onto the annulus

$$
A^{\prime}=\left\{z \in \mathbb{C}: e^{-1}|f(a)|<|z|<e|f(a)|\right\}
$$

and $V$ onto $A(|f(a)|)$. If $D(b, r)$ is a disk contained in $A(|f(a)|)$, then there is a branch of the logarithm mapping $D(b, r)$ into $Q_{\nu}(a)$ and thus a branch $\theta$ of the inverse of $f$ mapping $D(b, r)$ into $V$. Since $D(b, 2 r) \subset A^{\prime}$, the branch of the logarithm extends to a map from $D(b, 2 r)$ into $\Omega_{\nu}(a)$ and $\theta$ extends to a map from $D(b, 2 r)$ into $U$.

We now show that there are comparatively many disks disjoint $D(a, t)$ to which Lemma 5.1] can be applied.

Lemma 5.2. Let $\eta$ be as in Theorem 1.2. For sufficiently large $R$ there exist a positive integer $m(R)$ satisfying

$$
m(R) \geq \frac{\eta}{2}\left(\frac{R}{t(R)}\right)^{2}
$$

such that there are $m(R)$ points

$$
a_{j}=a_{j}(R) \in T\left(f, \alpha_{1}, \alpha_{2}, q, \lambda\right) \cap A(R), \quad j=1, \ldots, m(R),
$$

satisfying $D\left(a_{j}, t(R)\right) \subset A(R)$ for all $j$ and $D\left(a_{j}, t(R)\right) \cap D\left(a_{k}, t(R)\right)=\emptyset$ for $j \neq k$.

Proof. Let $m$ be the maximum number of points $a_{1}, \ldots, a_{m} \in T\left(f, \alpha_{1}, \alpha_{2}, q, \lambda\right) \cap A(R)$ which satisfy the conclusion of the lemma. Then

$$
T\left(f, \alpha_{1}, \alpha_{2}, q, \lambda\right) \cap A(R) \subset \bigcup_{k=1}^{m} D\left(a_{k}, 2 t(R)\right) \cup\{z \in A(R): \operatorname{dist}(z, \partial A(R)) \leq t(R)\},
$$

since a point contained in the left but not in the right side could be added to the collection $a_{1}, \ldots, a_{m}$. It follows that

$$
\text { area }\left(T\left(f, \alpha_{1}, \alpha_{2}, q, \lambda\right) \cap A(R)\right) \leq 4 \pi m t(R)^{2}+8 \pi R t(R) .
$$

Theorem 1.2 says that area $T\left(f, \alpha_{1}, \alpha_{2}, q, \lambda\right) \cap A(R) \geq 3 \pi \eta R^{2}$ and thus we obtain

$$
m \geq \frac{3 \pi \eta R^{2}-8 \pi R t(R)}{4 \pi t(R)^{2}} \geq \frac{\eta}{2}\left(\frac{R}{t(R)}\right)^{2}
$$

for large $R$.

For $m=m(R)$ and $a_{1}(R), \ldots, a_{m}(R)$ as in Lemma 5.2 we choose for each disk $D\left(a_{j}(R), t(R)\right)$ a subset $V$ as in Lemma 5.1. We denote these sets by $V_{1}(R), \ldots, V_{m}(R)$.

It follows that

$$
\operatorname{area}\left(\bigcup_{j=1}^{m(R)} V_{j}(R)\right) \geq m \beta t(R)^{2} \geq \frac{\eta}{2} \beta R^{2}
$$

Thus $\bigcup_{j=1}^{m(R)} V_{j}(R)$ has a positive density in $A(R)$. 
We now construct the sets $\mathcal{E}_{l}$ to which Lemma 4.4 will be applied. We choose $R_{0}$ large and put

$$
\mathcal{E}_{0}=\left\{A\left(R_{0}\right)\right\} \quad \text { and } \quad \mathcal{E}_{1}=\left\{V_{j}\left(R_{0}\right): 1 \leq j \leq m\left(R_{0}\right)\right\} .
$$

We shall define the sets $\mathcal{E}_{l}$ inductively such that if $F \in \mathcal{E}_{l}$, then $f^{l}(F)=A\left(R_{l, F}\right)$ for some $R_{l, F} \geq R_{0}$. Moreover, $f^{l-1}: F \rightarrow f^{l-1}(F)$ is bijective and if $G \in \mathcal{E}_{l-1}$ such that $F \subset G$, then

$$
f^{l-1}(F)=V_{j}\left(R_{l-1, G}\right) \subset D\left(a_{j}\left(R_{l-1, G}\right), t\left(R_{l-1, G}\right)\right)
$$

for some $j \in\left\{1, \ldots, m\left(R_{l-1, G}\right)\right\}$. To simplify notation, we will write $a_{j}$ instead of $a_{j}\left(R_{l-1, G}\right)$ in the sequel.

Suppose now that $\mathcal{E}_{l}$ has been defined and let $F \in \mathcal{E}_{l}$ and $G \in \mathcal{E}_{l-1}$ be as above. By Lemma 5.1 the disk $D\left(a_{j}, t\left(R_{l-1, G}\right)\right)$ has a subdomain $U$ which is mapped by $\log f$ bijectively onto $\Omega_{\nu}\left(a_{j}\right)$ for some $\nu \in\{1,2,3\}$, with $f^{l-1}(F)=V_{j}\left(R_{l-1, G}\right)$ being the subset that is mapped onto $Q_{\nu}\left(a_{j}\right)$. Thus $\log f^{l}: F \rightarrow Q_{\nu}\left(a_{j}\right)$ is bijective and its inverse $\psi: Q_{\nu}\left(a_{j}\right) \rightarrow F$ extends to $\Omega_{\nu}\left(a_{j}\right)$. For $1 \leq k \leq m\left(R_{l, F}\right)$ we can choose a domain $W_{k} \subset Q_{\nu}\left(a_{j}\right)$ such that $\exp W_{k}=V_{k}\left(R_{l, F}\right)$. We now put

$$
\mathcal{E}_{l+1}(F)=\left\{\psi\left(W_{k}\right): 1 \leq k \leq m\left(R_{l, F}\right)\right\} .
$$

Finally we set

$$
\mathcal{E}_{l+1}=\bigcup_{F \in \mathcal{E}_{l}} \mathcal{E}_{l+1}(F)
$$

Then the sequence $\left(\mathcal{E}_{l}\right)$ has the desired properties. Again we denote by $E_{l}$ the union of all elements of $\mathcal{E}_{l}$.

Lemma 5.3. There exists $\Delta>0$ such that if $F \in \mathcal{E}_{l}$, then

$$
\operatorname{dens}\left(E_{l+1}, F\right) \geq \Delta
$$

for all l. Moreover,

$$
\operatorname{diam} F \leq \exp \left(-l^{2}\right)
$$

for large $l$.

Proof. Let $F \in \mathcal{E}_{l}$ and let $\psi$ and $W_{k}$ be as above. Since $W_{k}=\log V_{k}\left(R_{l, F}\right)$ for some branch of the logarithm we have

$$
\operatorname{area} W_{k}=\int_{V_{k}\left(R_{l, F}\right)} \frac{1}{|z|^{2}} d x d y \geq \frac{1}{4 R_{l, F}^{2}} \text { area } V_{k}\left(R_{l, F}\right)
$$

so that (5.2) yields

$$
\operatorname{area}\left(\bigcup_{k=1}^{m\left(R_{l, F}\right)} W_{k}\right) \geq \frac{\eta \beta}{8} .
$$

Applying Lemma 4.1 we see that there exists a constant $C$ such that $\left|\psi^{\prime}(\zeta)\right| \leq C\left|\psi^{\prime}(z)\right|$ for $z, \zeta \in Q_{\nu}\left(a_{j}\right)$. (As the domains $\Omega_{\nu}\left(a_{j}\right)$ and the compact subsets $Q_{\nu}\left(a_{j}\right)$ are translates 
of fixed sets, the constant $C$ does not depend on $a_{j}$ or $\nu$.) Thus

$$
\begin{aligned}
\operatorname{dens}\left(E_{l+1}, F\right) & =\operatorname{dens}\left(\bigcup_{k=1}^{m\left(R_{l, F}\right)} \psi\left(W_{k}\right), \psi\left(Q_{\nu}\left(a_{j}\right)\right)\right) \\
& \geq \frac{1}{C^{2}} \operatorname{dens}\left(\bigcup_{k=1}^{m\left(R_{l, F}\right)} W_{k}, Q_{\nu}\left(a_{j}\right)\right) \\
& \geq \frac{\eta \beta}{32 C^{2} \pi \log 2} .
\end{aligned}
$$

Thus (15.3) holds with $\Delta=\eta /\left(32 C^{2} \pi \log 2\right)$.

To prove (5.4) let $F_{k} \in \mathcal{E}_{k}$ such that $F \subset F_{k}$, for $1 \leq k<l$. (With $G$ as before we thus have $G=F_{l-1}$.) With the abbreviation $R_{k}=R_{k, F_{k}}$ we have $f^{k}\left(F_{k}\right)=A\left(R_{k}\right)$. It follows from the construction and (1.5) that $R_{k+1} \geq R_{k}^{q}$ and thus $R_{k} \geq\left(R_{0}\right)^{q^{k}}$.

As before we have

$$
f^{l-1}(F)=V_{j}\left(R_{l-1}\right) \subset D\left(a_{j}, t\left(R_{l-1}\right)\right) \subset A\left(R_{l-1}\right)
$$

for some $j \in\left\{1, \ldots, m\left(R_{l-1}\right)\right\}$. Let $\phi$ be the branch of the inverse of $f^{l-1}$ which maps $f^{l-1}(F)$ to $F$. Noting that $\phi$ is univalent in $D\left(a_{j}, 2 t\left(R_{l-1}\right)\right)$ we deduce from Koebe's distortion theorem (i.e., Lemma 4.2) that if $z \in D\left(a_{j}, t\left(R_{l-1}\right)\right)$, then $\left|\phi^{\prime}(z)\right| \leq 12\left|\phi^{\prime}\left(a_{j}\right)\right|$. We conclude that

$$
\operatorname{diam} F \leq 12\left|\phi^{\prime}\left(a_{j}\right)\right| \operatorname{diam} f^{l-1}(F) \leq 24\left|\phi^{\prime}\left(a_{j}\right)\right| t\left(R_{l-1}\right) .
$$

Now $\left(f^{l-1}\right)^{\prime}(z)=\prod_{k=0}^{l-2} f^{\prime}\left(f^{k}(z)\right)$. Since $f^{k}(z) \in A\left(R_{k}\right)$ it follows from (5.1) that

$$
\left|f^{\prime}\left(f^{k}(z)\right)\right| \geq \gamma \frac{\left|f^{k+1}(z)\right|}{t\left(R_{k}\right)} \geq \gamma \frac{R_{k+1}}{t\left(R_{k}\right)}=\delta \frac{R_{k+1}}{R_{k}} \log M\left(R_{k}, f\right)
$$

where $\delta=\gamma \alpha_{1} /(2 \mu)$. We conclude that

$$
\left|\left(f^{l-1}\right)^{\prime}(z)\right| \geq \frac{R_{l-1}}{R_{0}} \prod_{k=0}^{l-2} \delta \log M\left(R_{k}, f\right) .
$$

Thus

$$
\left|\phi^{\prime}\left(a_{j}\right)\right| \leq \frac{R_{0}}{R_{l-1}} \prod_{k=0}^{l-2} \frac{1}{\delta \log M\left(R_{k}, f\right)}
$$

and hence

$$
\operatorname{diam} F \leq 24 \frac{R_{0}}{R_{l-1}} \prod_{k=0}^{l-2} \frac{1}{\delta \log M\left(R_{k}, f\right)} t\left(R_{l-1}\right)=\tau \prod_{k=0}^{l-1} \frac{1}{\delta \log M\left(R_{k}, f\right)}
$$

with $\tau=48 R_{0} \mu \delta / \alpha_{1}$. For large $R_{0}$ we have $\delta \log M(r, f) \geq \log r$ if $r \geq R_{0}$. Thus $\delta \log M\left(R_{k}, f\right) \geq \log R_{k} \geq q^{k} \log R_{0} \geq q^{k}$ if $R_{0}$ is chosen large enough. Hence

$$
\operatorname{diam} F \leq \tau \prod_{k=0}^{l-1} q^{-k}=\tau \exp \left(-\frac{1}{2}(l-1) l \log q\right) \leq \exp \left(-l^{2}\right)
$$

for large $l$, since we have chosen $q=8>e^{2}$. 
Lemma 5.3 says that we can apply Lemma 4.4 with

$$
\Delta_{l}=\Delta \quad \text { and } \quad d_{l}=\exp \left(-l^{2}\right) .
$$

This yields $\operatorname{dim} E=2$. Moreover, it follows from the construction that $E \subset I(f)$. By Lemma 4.5 we have $A(R) \cap J(f) \neq \emptyset$ for large $R$. This implies that $F \cap J(f) \neq \emptyset$ if $F \in \mathcal{E}_{l}$ and if $l$ is sufficiently large. Hence $E \subset J(f)$. Altogether we thus have $E \subset I(f) \cap J(f)$. This completes the proof of Theorem 1.1.

\section{REFERENCES}

[1] M. Aspenberg and W. Bergweiler, Entire functions with Julia sets of positive measure. Preprint, arxiv: 0904.1295.

[2] K. Barański, Hausdorff dimension of hairs and ends for entire maps of finite order. Math. Proc. Cambridge Philos. Soc. 145 (2008), 719-737.

[3] K. Barański, B. Karpińska and A. Zdunik, Hyperbolic dimension of Julia sets of meromorphic maps with logarithmic tracts. Int. Math. Res. Not. 2009 (2009), 615-624.

[4] W. Bergweiler, Iteration of meromorphic functions. Bull. Amer. Math. Soc. (N. S.) 29 (1993), 151188.

[5] W. Bergweiler, B. Karpińska and G. M. Stallard, The growth rate of an entire function and the Hausdorff dimension of its Julia set. To appear in J. London Math. Soc., arXiv: 0807.2363.

[6] A. E. Eremenko and M. Yu. Lyubich, Dynamical properties of some classes of entire functions. Ann. Inst. Fourier 42 (1992), 989-1020.

[7] P. Fatou, Sur l'itération des fonctions transcendantes entières. Acta Math. 47 (1926), 337-360.

[8] A. J. Macintyre and W. H. J. Fuchs, Inequalities for the logarithmic derivatives of a polynomial. J. London Math. Soc. 15 (1940), 162-168.

[9] A. A. Goldberg and I. V. Ostrovskii, Value distribution of meromorphic functions. Transl. Math. Monographs 236, American Math. Soc., Providence, R. I., 2008.

[10] W. K. Hayman, Meromorphic functions. Clarendon Press, Oxford, 1964.

[11] W. K. Hayman, On the characteristic of functions meromorphic in the plane and of their integrals. Proc. London Math. Soc. (3) 14a (1965), 93-128.

[12] B. Ja. Levin, Distribution of zeros of entire functions. American Mathematical Society, Providence, R. I., 1964

[13] C. McMullen, Area and Hausdorff dimension of Julia sets of entire functions. Trans. Amer. Math. Soc. 300 (1987), 329-342.

[14] J. Miles and J. Rossi, Linear combinations of logarithmic derivatives of entire functions with applications to differential equations. Pacific J. Math. 174 (1996), 195-214.

[15] L. Rempe, Rigidity of escaping dynamics for transcendental entire functions. To appear in Acta Math., arXiv: math/0605058.

[16] G. Rottenfußer, J. Rückert, L. Rempe and D. Schleicher, Dynamic rays of bounded-type entire functions. To appear in Ann. of Math., arXiv: 0704.3213.

[17] H. Schubert, Über die Hausdorff-Dimension der Juliamenge von Funktionen endlicher Ordnung. Dissertation, University of Kiel, 2007; http://eldiss.uni-kiel.de/macau/receive/ dissertation_diss_00002124.

[18] G. M. Stallard, The Hausdorff dimension of Julia sets of entire functions II. Math. Proc. Cambridge Philos. Soc. 119 (1996), 513-536.

[19] G. M. Stallard, Dimensions of Julia sets of transcendental meromorphic functions, in "Transcendental Dynamics and Complex Analysis". London Math. Soc. Lect. Note Ser. 348. Edited by P. J. Rippon and G. M. Stallard, Cambridge Univ. Press, Cambridge, 2008, pp. 425-446.

[20] M. Taniguchi, Size of the Julia set of structurally finite transcendental entire function. Math. Proc. Cambridge Philos. Soc. 135 (2003), 181-192.

[21] D. Townsend, Comparisons between $T(r, f)$ and the total variation of $\arg f\left(r e^{i \theta}\right)$ and $\log \left|f\left(r e^{i \theta}\right)\right|$. J. Math. Anal. Appl. 128 (1987), 347-361. 
[22] G. Valiron, Lectures on the general theory of integral functions, Édouard Privat, Toulouse, 1923; Chelsea, New York, 1949.

[23] J.-H. Zheng, On multiply-connected Fatou components in iteration of meromorphic functions. J. Math. Anal. Appl. 313 (2006), 24-37.

Mathematisches Seminar, Christian-Albrechts-Universität zU Kiel, Ludewig-MeynStr. 4, D-24098 Kiel, Germany

E-mail address: bergweiler@math.uni-kiel.de

Faculty of Mathematics and Information Science, Warsaw University of Technology, Pl. Politechniki 1, 00-661 Warszawa, Poland

E-mail address: bkarpin@mini.pw.edu.pl 